\title{
Investigating the fatigue behavior of grain-oriented Fe-3\%Si steel sheets using magnet-optical Kerr microscopy and micromagnetic multiparameter, microstructure and stress analysis
}

\author{
Shayan Deldar ${ }^{1, *}$, Marek Smaga ${ }^{1}$, and Tilmann Beck $^{1}$ \\ ${ }^{1}$ University of Kaiserslautern, Institute of Materials Science and Engineering, Kaiserslautern, Germany
}

\begin{abstract}
Fatigue is considered as a reason for a significant number of mechanical failures of engineering materials. Conventionally, microstructural investigations along with stress-strain hysteresis measurements are performed to understand and characterize the fatigue behavior of metallic materials. Moreover, further physical data like temperature, electrical resistance and, in the case of ferromagnetic materials, magnetic properties can be used for a comprehensive characterization of fatigue process. The present work has employed Magneto-Optical Kerr Effect (MOKE) microscope and Micromagnetic Multiparameter, Microstructure and stress Analysis (3MA) system to illustrate magnetic domain structure and various intrinsic magnetic properties including magnetic Barkhausen noise (MBN) of the investigated material. In order to investigate the influence of the mechanical deformation processes on the magnetic parameters, samples were produced out of the grain-oriented electrical steel sheets and were subjected to a tensile test as well as a cyclic strain increase load test with $\mathrm{R}=0$ at ambient temperature.
\end{abstract}

\section{Introduction}

Although the magneto-mechanical behavior of ferromagnetic materials has been investigated for almost 150 years [1], a detailed understanding and visualization of the underlying phenomena caused by plastic deformation and fatigue process is yet to be elaborated. Electrical steels are considered as one of the conventional group of ferromagnetic materials well known to the magnetic community [2]. Their coarse magnetic domain structure, high magnetic flux density, and low iron loss have made this group of ferromagnetic materials suitable for usage in internal permanent magnet (IPM) motor cores [3, 4]. Developments in the automobile industry focusing on electric vehicles (EV) and hybrid electric vehicles (HEV) as well as efforts to increase the fuel efficiency of internal combustion engines resulted in a progressively increasing demand for research and development on this material class in the recent years [3]. Since the rotor core of an IPM motor is subjected to centrifugal forces which undergo fluctuations due to variations in the rotational speed of the motor during operation, the electrical steels used for this application not only should have acceptable mechanical properties, but also must maintain their magnetic characteristics in order to ensure a durable and efficient electrically powered system.

As a result, investigations on electrical steels recently directed to their magneto-mechanical behavior, fatigue properties, manufacturing technologies, and advanced magnetic measurements on the two main groups of electrical steels, grain-oriented and non-oriented [1, 511]. Perevertov et al. $[1,5]$ described magnetic domain structure and hysteresis loops of grain-oriented $\mathrm{Fe}-3 \% \mathrm{Si}$ under the influence of tensile and compressive elastic stresses. Fatigue behavior of high grade non-oriented electrical steels was published by Liying et al. [9] focusing on crack characterization and fracture mechanisms as well as the Wöhler curve determined for a specific batch of non-oriented electrical steel (30WGP1600). Furthermore, Dehmani et al. [6, 7] investigated M300-35A non-oriented electrical steels taking into account the effect of the punching process and annealing as parts of the manufacturing procedures. The negative effect of punching and positive effects of mechanical polishing on the fatigue properties of nonoriented electrical steel has been demonstrated. The article also has focused on the microstructure and fracture surface morphology. One of the few works regarding the influence of the fatigue loading on the magnetic properties of electrical steels has been presented by Karthaus et al. [8] demonstrating the changes of the magnetic polarization hysteresis curve at different numbers of cycles as well as a comparison between fatigue tests with different maximum nominal stress amplitudes. In that study, fatigue-induced cracking of the specimen was not investigated.

In contrast to this, the present work aims to characterize the influences of mechanical strains on the magnetic properties of grain-oriented electrical steels 
with 3 wt.- $\%$ silicone content. The mechanical strain was applied in a stepwise strain controlled tensile. The correlation between mechanical loading, magnetic domain structure visualized by Magneto-Optical Kerr Effect (MOKE) and integral magnetic properties measured via a Micromagnetic Multiparameter, Microstructure and stress Analysis (3MA) system are discussed. A comprehensive analysis of the magnetic domain structure and magnetic Barkhausen noise (MBN) signal measured at the loaded and unloaded steps is presented.

\section{Investigated material}

The material investigated in this work is a "powercore" grain-oriented electrical steel. It holds a $\{110\}<001>$ (Figure 1) texture along the rolling direction. This socalled Goss texture [5] is typical in grain-oriented electrical steel and effects magnetic anisotropy with large magnetic flux density in the rolling direction (RD). The chemical composition of the investigated material is shown in Table 1.

Table 1. The chemical composition of the investigated grainoriented electrical steel.

\begin{tabular}{|c|c|c|c|c|}
\hline $\begin{array}{c}\text { Chemical } \\
\text { composition }\end{array}$ & C & Mn & Si & Fe \\
\hline In wt.-\% $\%$ & 0.003 & 0.070 & 3.000 & Bal. \\
\hline
\end{tabular}

The mentioned texture of the material is schematically illustrated in figure 1(a). tensile specimens were cut out of the steel sheet with the geometry shown in figure 1(b) and longitudinal axes oriented in RD. The mechanical stress and magnetic field are applied parallel to the rolling direction (RD).

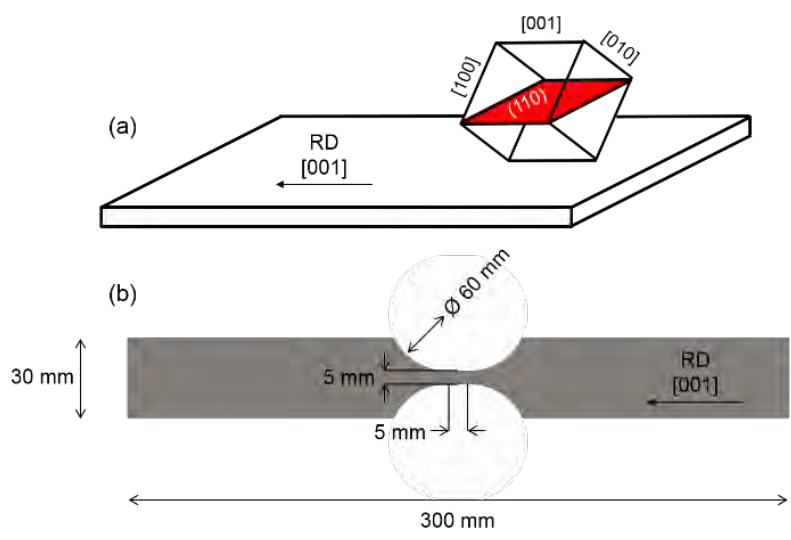

Fig. 1. Schematic of the Goss texture longitudinal to the rolling direction (a) and the specimen geometry (b).

The mechanical properties of the investigated material are summarized in table 2. In comparison to 30WGP1600 and M300-35A steels investigated respectively in $[7,9]$, the grain-oriented electrical steel studied in this work exhibits significantly lower yield stress and tensile strength. Note that the crystallographic texture of the grain-oriented electrical steel results in a variation of the mechanical properties with respect to the measurement direction while in case of non-oriented steels, the material has isotropic properties.

Table 2. Mechanical properties of the investigated grainoriented electrical steel.

\begin{tabular}{|c|c|}
\hline Description & Value \\
\hline Thickness & $0.27 \mathrm{~mm}$ \\
\hline Yield stress & $300 \mathrm{MPa}$ \\
\hline Tensile strength & $340 \mathrm{MPa}$ \\
\hline Elongation to fracture & $10 \%$ \\
\hline Hardness & $175-195 \mathrm{HV} 0.1$ \\
\hline
\end{tabular}

\section{Experimental setup}

Before the experimental measurements, the samples were mechanically ground up to 4000 grit $\mathrm{SiC}$ paper in the deformation area of the specimen and subsequently polished on a cotton cloth in $3 \mu \mathrm{m}$ diamond suspension.

\subsection{Magneto-optical Kerr microscope and tensile test system for in-situ loading}

For visualization of the magnetic domain structure, a magneto-optical Kerr microscope was employed in longitudinal mode. Figure 3 shows the experimental setup and a schematic overview of its components. Magneto-Optical Kerr Effect (MOKE) enables a regular polarization optical microscope to be used for magnetic domain and domain wall observation [12]. The working principle of the Kerr microscope is based on the application of linearly polarized light on the surface of the magnetic material. Due to the physics of Lorenz force when the linearly polarized light reflects from the surface of a ferromagnetic material, the plane of polarization is rotated because of the surface magnetization. The rotation of the polarization plane differs for the lights reflected from regions with different magnetizations. Therefore, the reflected light obtains independent polarization planes with respect to the regions from where they reflected. The reflected light with different polarization directions are the indicators of the magnetic domain contrast on the surface of the ferromagnetic material. To obtain a high signal to noise ratio and eliminate the negative effect of surface roughness of the specimen on the magnetic domain image quality, and to enhance image contrast differential imaging technique was used. In this method, two images are taken at two different magnetic stages of the hysteresis, one of which is usually the saturation state. The two images are then digitally subtracted from each other and as a result, topographic effects of the surface 
are eliminated and only the difference between the magnetic domain structures appears [2]. For the differential imaging to be successful, the two images must be at the same spatial position with high precision. In order to have a fixed position of the sample while differential imaging, a piezo stabilization system has been applied underneath the sample stage which immediately corrects the drifts which always occur due to the magnetic force on the ferromagnetic material under investigation.

In the schematic sketch, it is demonstrated that the illumination path of the light beam is applied to the surface at a certain angle (angle of incidence). This angle is necessary for the polarization rotation to occur. The larger the angle of incidence, the higher the raw image contrast. Since both, the illumination path and the reflection path (observation path), go through the objective lens of the Kerr microscope, one has to take into account for selection of the proper objective lens for MOKE application.

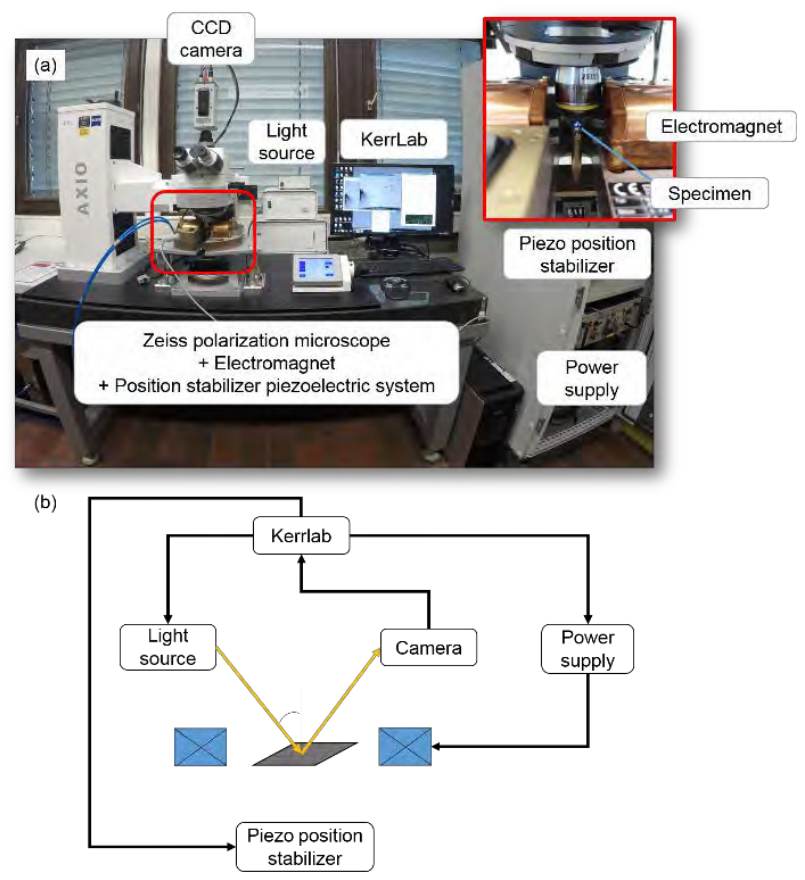

Fig. 2. Magneto-optical Kerr effect (MOKE) experimental setup (a) and a schematic sketch of the setup (b).

The tensile test system shown in figure 3 has been designed specifically for application as an in-situ tensile module for Kerr microscopy. The window placed in between the two electromagnets and the geometry of the electromagnets allow the objective lens to reach the working distance to the specimen. The electromagnets apply the magnetic field parallel to the loading direction. The setup is, in fact, capable of performing an elongation controlled tensile loading since the loading is done via turning the tension screw clockwise. The load cell then monitors the tension applied by the tension screw. As the device limits the force applied to a maximum of $1 \mathrm{kN}$ the specimen geometry (figure 1(b)) was designed appropriately.
Because of the large size of the specimen, the preparation and polishing of the surface were challenging. Once the surface quality of the specimen in the deformation area is proper, magnetic domain observation during the application of the tension was possible.

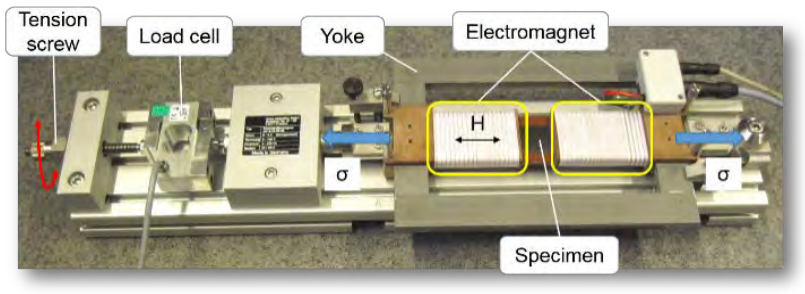

Fig. 3. The in-situ tensile test designed for Kerr microscope.

\subsection{MA sensor}

Figure 4(a) shows a Micromagnetic Multiparameter, Microstructure and stress Analysis (3MA) system which is classified as a non-destructive evaluation device. The parameters obtained from this setup are used for online monitoring of e.g. machinery components and steel production processes. With this setup, only ferromagnetic materials can be characterized by their integral magnetic properties such as magnetic Barkhausen noise, incremental permeability, and harmonic analysis of the tangential magnetic field. As the magnetic properties experience variations under the influence of mechanical deformation, the 3MA system was used to record the corresponding magnetic behavior. $[13,14]$ In the present study, the focus is on the analysis of the magnetic Barkhausen noise signal obtained from the $3 \mathrm{MA}$ sensor.

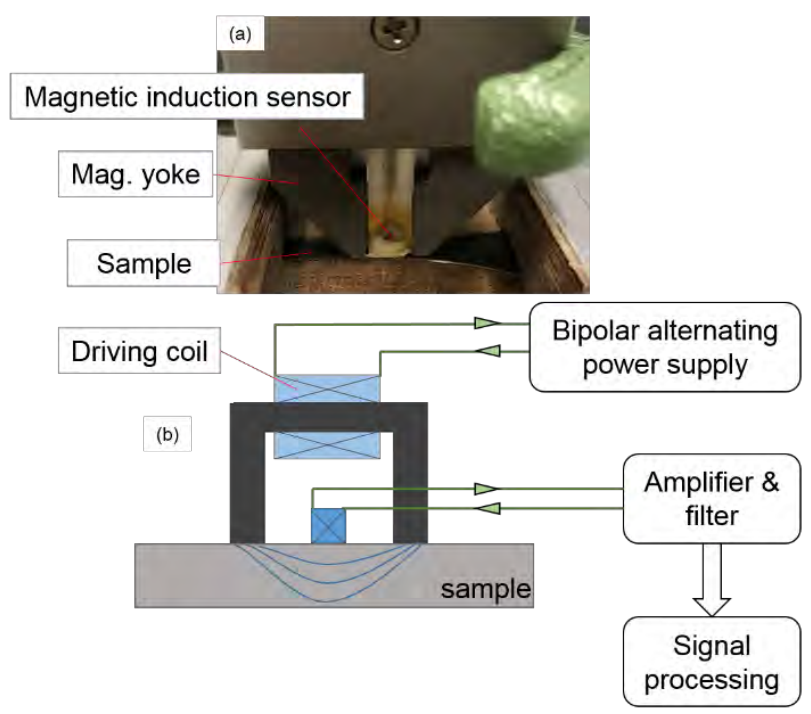

Fig. 4. Photograph of the $3 \mathrm{MA}$ sensor (a) and a schematic sketch of the 3MA system (b).

Magnetic Barkhausen noise is a physical property of magnetic materials which is a result of Bloch wall 
motion during the magnetization reversal process. A ferromagnetic material in the demagnetized state experiences a multi-domain magnetic microstructure, such that the material is, from the thermodynamical point of view, at its lowest total free energy [2]. As the material is applied to the external magnetic field, this multi-domain magnetic microstructure converts during a so-called magnetic reversal process to a single domain magnetization (saturation magnetization). The reversal process includes domain wall movement and rotation of magnetization. The domain wall movement mechanism is not a continuous process and, because in iron-based ferromagnetic materials Bloch walls are present, this mechanism is also called Bloch wall jumps [13]. The reason for these discontinuities in the domain wall movements is microstructural obstacles within the path of the domain walls by which the Bloch walls are pinned. Grain boundaries, dislocations, and precipitations are examples of the above mentioned microstructural obstacles. The pick-up coil (magnetic induction sensor in figure 4(b)) directly receives the Barkhausen noise and the data acquisition system converts it to an electrical voltage.

In order to induce an alternating magnetic field to the specimen, a bipolar alternating power supply is connected to a driving coil and the magnetic induction generated by the driving coil is transferred to the specimen by a high permeability magnetic core and the magnetic response of the specimen is read out by the pick-up coil. The signal is then amplified and analyzed.

\section{Results and discussion}

Figure 5(a) illustrates the grain structure of the investigated material which indicates an average grain size of $10 \mathrm{~mm}$. Besides the crystallographic grain structure observable at the surface of the material, the coexistence of the magnetic domain structure in the undeformed state of the grain-oriented electrical steel and its interaction with the crystallographic orientation is pictured. Moreover, it is shown that because of the crystallographic texture of the material parallel to the rolling direction, a magnetocrystalline anisotropy exists in the material leading to the magnetization direction along the rolling direction. In the magnetic domain image, one can notice a disturbance of the domain structure in the grain on the left side of figure 5(b) compared to the grain on the right side of the image. The reason for such a magnetic structure difference is a small degree of misorientation in the left grain with respect to the right grain.

Figure 6(a) illustrates a cyclic strain increase load test performed by the in-situ tensile testing device (figure 3 ). The test with a strain ratio of $\mathrm{R}_{\varepsilon}=0$ was performed by loading and unloading the specimen repeatedly until the specimen failure. By each loading step, $1.7 \%$ additional plastic strain was applied to the specimen and the measured force was recorded. After each loading step, the elastic strain was completely released by reducing the tensile force to zero. For each loading and unloading step in the plastic region (points $\mathrm{c}$ to $\mathrm{g}$ and $\mathrm{c}$ ' to g'), longitudinal magnetic domain image was captured (figure 7) and maximum magnetic Barkhausen noise (MBN) signal within one hysteresis loop was extracted (figure 6(b)).
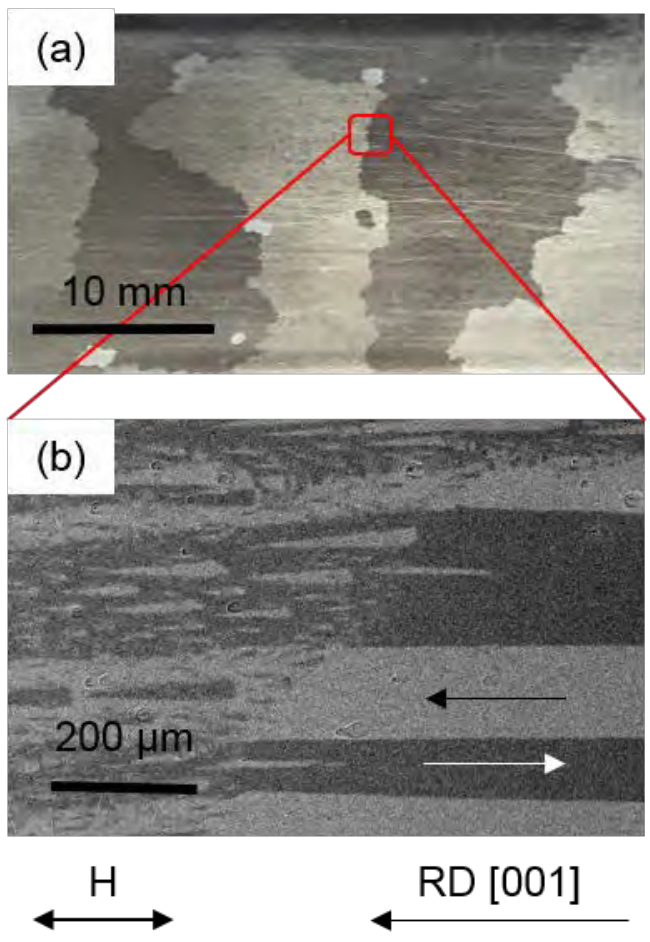

Fig. 5. Crystallographic microstructure (a) and magnetic domain structure (b) of the grain-oriented electrical steels at the initial state.

The blue and red lines in figure 6 indicate the loaded and unloaded condition in the strain increase load test. The dashed blue lines illustrate each loading/unloading step (for instance unloading from d to d' and loading from d' to d). Based on the behavior of the maximum MBN in the loaded condition (the blue line in figure 6(b)) the diagram was divided into 3 zones. At Zone 1 which was still in the elastic region, the maximum Barkhausen noise at the loaded state with respect to the initial state decreased. This means the stress-induced magnetic anisotropy helped the magnetization reversal by decreasing the energy required for domain wall unpinning as a driving force. At zone 2, where the plastic deformation started, the maximum MBN reached a minimum and began to rise at about $6 \%$ total strain. The beginning of the plastic regime is where the dislocation density started to increase, in other words, more pinning sources were generated. Therefore, a contribution in favor of MBN increase is produced. Before zone 3, the sample has already experienced $4 \%$ plastic deformation, therefore the mechanisms which resulted in MBN increase were already dominant. This argument is also valid when considering the maximum $\mathrm{MBN}$ curve at the unloaded condition (red curve in figure 6 (b)) where the MBN showed a steady increase throughout the strain 
increase load test because, in the unloaded condition, stress-induced effects did not assist the magnetization reversal by easing the domain wall movement.

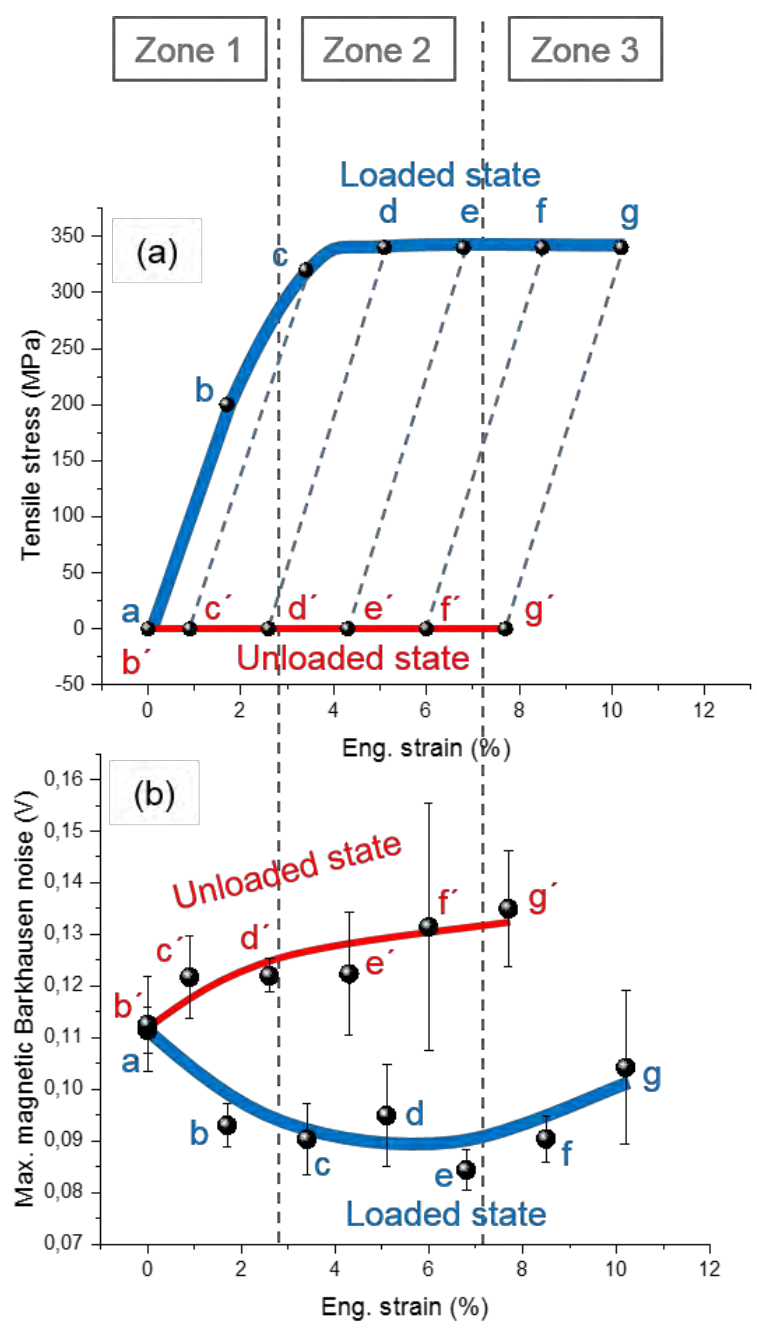

Fig. 6. Nominal stress vs. engineering strain curve of the cyclic strain increase load test performed by the in-situ testing device (a) and maximum magnetic Barkhausen noise measurement for each step in loaded and unloaded condition (b).

The MOKE measurements were done in the longitudinal mode. The applied magnetic field, the uniaxial magnetic anisotropy, the sensitivity direction (which is defined by the illumination path), the applied mechanical stress, and the rolling direction in figure 7 are all horizontally directed. The magnetic domain images in figure 7 indicate that domain refinement occurs by application of strain in both, loaded and unloaded condition. Furthermore, a comparison between the respective images in the loaded and unloaded condition in each step shows that the magnetic domain structure at the unloaded state tends to exhibit a higher domain wall density, i.e. finer domain structure. This correlates with figure 6(b) indicating higher maximum MBN in the unloaded (red curve) than in the loaded state (blue curve), i.e. the magnetic Barkhausen noise of the unloaded condition is always higher than that in the respective loaded condition.
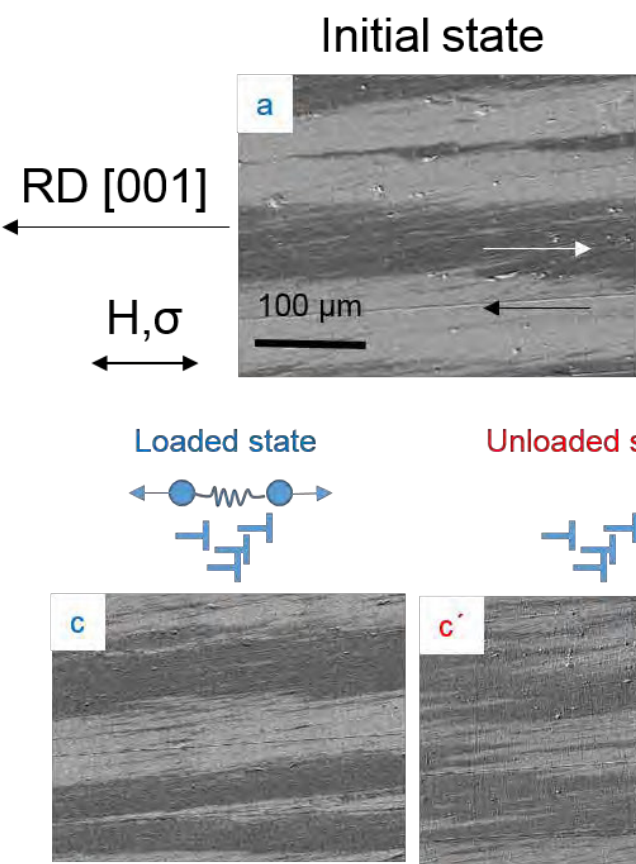

Unloaded state
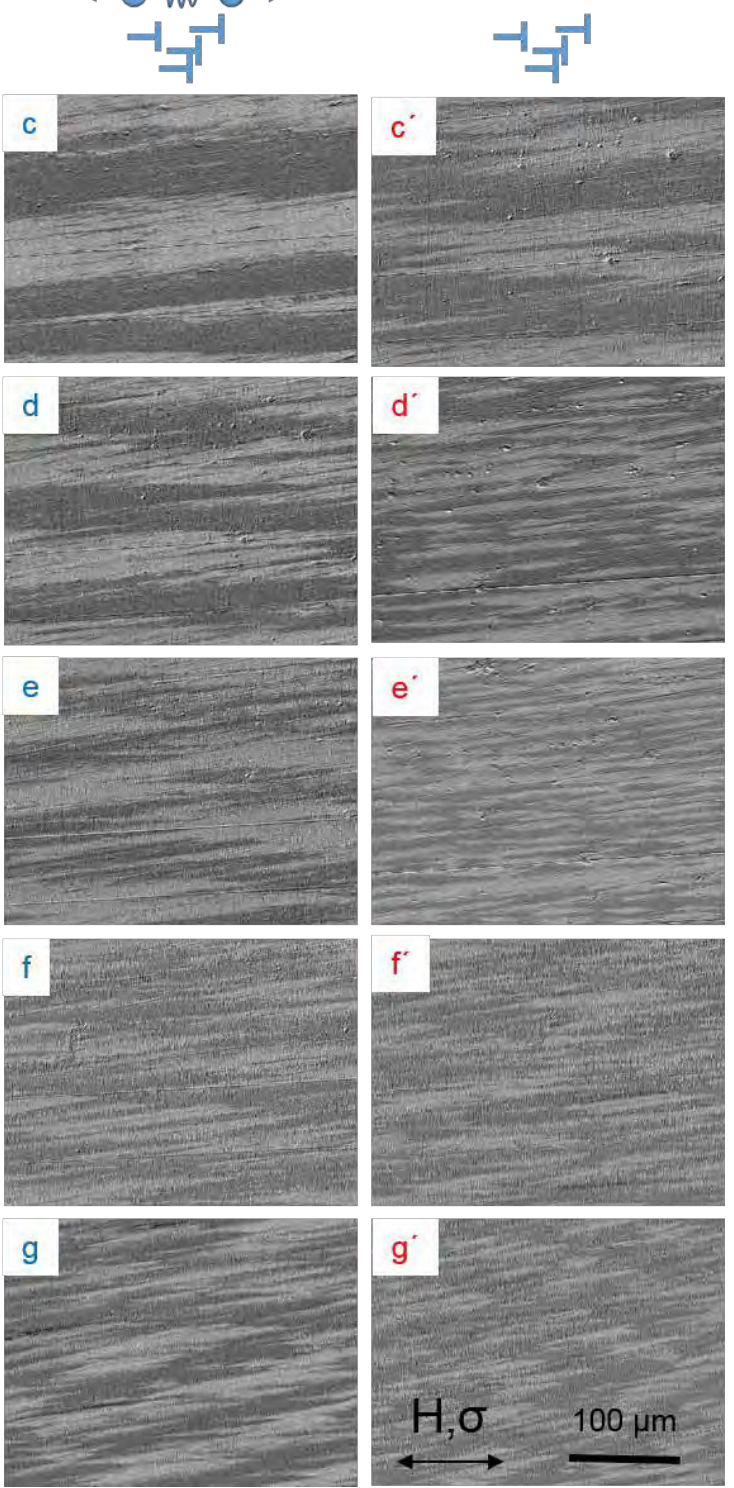

Fig. 7. Magnetic domain images at the states of the strain increase load test indicated in Figure 6.

\section{Summary and conclusion}


Cyclic strain increase tests with a strain ratio $R_{\varepsilon}=0$ were performed to investigate the influence of the mechanical deformation processes on the magnetic domain structure and integral magnetic parameters. Magneto-Optical Kerr Effect (MOKE) microscopy and a Micromagnetic Multiparameter, Microstructure and stress Analysis (3MA) system were employed to illustrate magnetic domain structure and intrinsic magnetic properties including magnetic Barkhausen noise (MBN). The tensile test system by which the cyclic strain increase load test was performed was specifically designed for application as an in-situ tensile module for Kerr microscope. Presence of the stress-induced anisotropy acted as a driving force for domain wall movement which led to decrease of the maximum $\mathrm{MBN}$ in the elastic regime at the loaded condition. As the dislocation density increased during plastic deformation, the maximum MBN in the loaded state started to increase. The magnetic domains showed a refined structure with increasing plastic strain applied during cyclic strain increase load testing. The domain structures at the unloaded condition indicated higher domain wall densities compares to the loaded condition at the same plastic strain which is in agreement with the MBN curves.

\section{Acknowledgment}

This research was supported by German Research Foundation (DFG) within the Transregional Collaborative Research Center SFB/TRR 173 (Spin+X), project B08. We would also like to show our gratitude to Prof. Rudolf Schäfer for sharing his experiences concerning MOKE experimental setup.

\section{References}

1. O. Prevertov, R. Schäfer, J. Phys. D: Appl. Phys. 47, 185001 (2014)

2. A. Hubert, R. Schäfer, Magnetic Domains, $3^{\text {rd }}$ edition (Heidelberg: Springer) (1998)

3. Y. Oda, M. Kohno, A. Honda, JMMM 320, 24302435 (2008)

4. I. Tanaka, H. Nitomi, K. Imanishi, K. Okamura, H. Yashiki, IEEE Transactions on Magnetics, 49, 2297 3001 (2013)

5. O. Prevertov, R. Schäfer, J. Phys. D: Appl. Phys. 45, 135001 (2012)

6. H. Dehmani, C. Brugger, T. Palin-Luc, C. Mareau, S. Koechlin, Procedia Engineering, 133, 556-561 (2015)

7. H. Dehmani, C. Brugger, T. Palin-Luc, C. Mareau, S. Koechlin, Procedia Structural Integrity, 2, 32563263 (2016)

8. J. Karthaus, S. Steentjes, D. Gröbel, K. Andreas, M. Merklein, K. Hameyer, Archives of Electrical Engineering, 66(2), 351-360 (2017)
9. D. Liying, Z. Guifeng, L. Jing, S. Wenmin, B. Yunjie, C. Zhaoyang, x. Huan, Journal of Wuhan University of Technology-Mater. Sci. Ed., 32, 13291335 (2017)

10. M. Petrun, S. Steentjes, K. Hameyer, B. Polajzer, IEEE Transactions on Magnetics, 53, 1-5 (2017)

11. N.J. Lewis, P.I. Anderson, Y. Gao, F. Robinson, JMMM, 452, 495-501 (2018)

12. J. McCord, J. Phys. D: Appl. Phys. 48, 333001 (2015)

13. G. Dobmann, Physical Basics and Industrial Applications of $3 M A \quad-\quad$ Micromagnetic Multiparameter Microstructure and Stress Analysis, (IOS Press publishing house) (2007)

14. G. Hübschen, I. Alpeter, R. Tschuncky, H. Herrmann, Materials Characterization Using Nondestructive Evaluation (NDE) Methods, (Elsevier) (2016) 